\title{
Trends in Participation Rates for the National Cancer Screening Program in Korea, 2002-2012
}

Mina Suh, MD, $\mathrm{PhD}^{1}$
Seolhee Song, $\mathrm{BA}^{1}$
Ha Na Cho, $\mathrm{BN}^{2}$
Boyoung Park, MD, $\mathrm{PhD}^{2}$
Jae Kwan Jun, MD, $\mathrm{PhD}^{1}$
Eunji Choi, MPH²
Yeol Kim, MD, MPH ${ }^{1}$
Kui Son Choi, $\mathrm{PhD}^{2}$

${ }^{1}$ National Cancer Control Institute, National Cancer Center, Goyang, ${ }^{2}$ Graduate School of Cancer Science and Policy, National Cancer Center, Goyang, Korea
Correspondence: Kui Son Choi, PhD Graduate School of Cancer Science and Policy, National Cancer Center, 323 Ilsan-ro, Ilsandong-gu, Goyang 10408, Korea Tel: 82-31-920-2912

Fax: 82-31-920-2189

E-mail: kschoi@ncc.re.kr

Received May 2, 2016

Accepted October 21, 2016

Published Online November 11, 2016

\section{Purpose}

The National Cancer Screening Program (NCSP) in Korea supports cancer screening for stomach, liver, colorectal, breast, and cervical cancer. This study was conducted to assess trends in participation rates among Korean men and women invited to undergo screening via the NCSP as part of an effort to guide future implementation of the program in Korea.

\section{Materials and Methods}

Data from the NCSP for 2002 to 2012 were used to calculate annual participation rates with 95\% confidence intervals $(\mathrm{Cl})$ by sex, insurance status, and age group for stomach, liver, colorectal, breast, and cervical cancer screening.

\section{Results}

In 2012, participation rates for stomach, liver, colorectal, breast, and cervical cancer screening were $47.3 \%, 25.0 \%, 39.5 \%, 51.9 \%$, and $40.9 \%$, respectively. The participation rates increased annually by $4.3 \%(95 \% \mathrm{Cl}, 4.0$ to 4.6$)$ for stomach cancer, 3.3\% (95\% Cl, 2.5 to 4.1) for liver cancer, $4.1 \%(95 \% \mathrm{Cl}, 3.2$ to 5.0$)$ for colorectal cancer, $4.6 \%$ (95\% Cl, 4.1 to 5.0) for breast cancer, and $0.9 \%(95 \% \mathrm{Cl},-0.7$ to 2.5$)$ for cervical cancer from 2002 to 2012.

\section{Conclusion}

Participant rates for the NCSP for the five above-mentioned cancers increased annually from 2002 to 2012.

\section{Introduction}

Cancer is a leading cause of death, and cancer burden is expected to grow worldwide due to aging populations. For 2012, GLOBOCAN reported 14.1 million new cancer cases and 8.2 million deaths from cancer worldwide [1]. In Korea, cancer has remained the leading cause of death since 1983, when statistics first began to be collected [2]. Over the past 14 years, overall incidence rates have increased by $3.3 \%$ per
Key words

Neoplasms, Early detection of cancer, Mass screening year, and cancer is now responsible for nearly one in four deaths [3]. In 2012, a total of 224,177 cancer cases and 73,759 cancer deaths were reported in Korea, with stomach, liver, and colorectal cancers accounting for $45 \%$ of all newly diagnosed cancers in men, while stomach, liver, colorectal, breast, and cervical cancers accounted for $41 \%$ of all newly diagnosed cancers in women [3].

To reduce the cancer burden the World Health Organization (WHO) has suggested implementation of National Cancer Control Plans for prevention, early detection, diagnosis, 
treatment, and palliation [4]. In 1996, the Korean government initiated a comprehensive "10-year National Cancer Control Plan (10-yr NCCP)" [5]. As part of this plan, the National Cancer Screening Program (NCSP) was launched in 1999. Currently, Korean men and women older than 40 years are eligible for stomach cancer screening via endoscopy or upper gastrointestinal series biennially. Liver cancer screening is only provided to people aged 40 years and over who are hepatitis B surface antigen or anti-hepatitis $\mathrm{C}$ virus (HCV) positive or have liver cirrhosis. An ultrasonographic examination and $\alpha$-fetoprotein test is offered every 6 months for these high risk groups. Colorectal cancer screening is conducted for individuals aged 50 years and older, primarily via an annual fecal occult blood test (FOBT). People with positive results from the FOBT can choose to undergo either colonoscopy or a double-contrast barium enema test, as well as a histological examination if needed. Mammography is provided biannually to women aged 40 years or over. Every 2 years, a Pap smear is provided to women aged 30 years and over for cervical cancer screening [6].

Organized screening programs must be able to ensure high coverage and participation, as adequate participation in screening is essential to reducing cancer mortality [7]. Upon evaluation of the progress made by the first 10-yr NCCP from 1996 to 2005, the Korean Ministry of Health and Welfare developed plans for a second 10-yr NCCP for 2006-2015. The second 10-yr NCCP was designed to improve cancer screening rates among all Koreans by improving quality of screening and expanding support for cancer patients. The 10-year plan also sought to increase participation rates for the NCSP to 55\% by 2015 [8]. Accordingly, the present study aimed to examine trends in participation rates in stomach, liver, colorectal, breast, and cervical cancer screening via the NCSP from 2002 to 2012. We also attempted to evaluate how NCSP policy changes affected these rates.

\section{Materials and Methods}

The data used in this study were collected from the NCSP database for 2002 to 2012. The NCSP database includes information on age, sex, and type of health insurance (Medical Aids, National Health Insurance) for individuals who were invited to undergo screening for stomach, liver, colorectal, breast, and cervical cancer via the NCSP. The database also includes information on screening date and screening results (negative, suspicious, highly suggestive of malignancy, or benign) for those who participated in the NCSP.

In the NCSP, all eligible men and women receive an invitation letter, along with information on screening methods and the locations of screening units, from the National Health Insurance Service (NHIS), beginning in January of each year. The NHIS selects eligible men and women for each cancer site according to the NCSP protocol (S1 Table). For liver cancer screening, the NHIS defines high-risk individuals as those who have been tested or received medical care for hepatitis B virus or HCV infection, chronic hepatitis, chronic liver disease, or liver cirrhosis within the past 2 years.

In this study, participation rates were analyzed on a single-year basis between the years of 2002 and 2012, and assessed as the percentage of eligible people who underwent screening among those invited. The participation rates for each of the five major cancers were calculated as the percentage of people who participated in each cancer screening program among those invited by the NCSP to undergo screening according to the NCSP protocol (S1 Table). Participation rates for each of the five cancers were also calculated according to sex, age, and health insurance status. We used health insurance status as a proxy for socioeconomic status. Insurance status was classified into one of three categories: medical aids program (MAP) recipients (extremely poor people who received livelihood assistance and were unable to pay for health care or insurance), NHIS beneficiaries of lowincome status (target population for free-of-charge screening), and NHIS beneficiaries of high income status (target population for screening with a copayment).

To estimate changes in participation rates, we assessed the average annual percentage change (APC) by comparing rates for 2002 and 2012 as relative rates. These risks were reported as the average APC ([relative risk-1] $\times 100 /$ number of years) with 95\% confidence intervals (CIs). All data were analyzed using the SAS statistical software ver. 9.3 (SAS Inc., Cary, NC). This study was approved by the Institutional Review Board of the National Cancer Center in Korea (approval number: NCCNCS-08-129).

\section{Results}

The trends in participation rates in the stomach, liver, colorectal, breast, and cervical cancer screening via the NCSP from 2002 to 2012 are shown in Fig. 1. The number of men and women invited to undergo stomach cancer screening increased from 9.8 million in 2002 to 12.6 million in 2012. During this period, the NCSP supported 37.6 million examinations for stomach cancer. Stomach cancer screening participation rates increased from $7.5 \%$ in 2002 to $47.3 \%$ in 2012 (Table 1). Individuals aged 60 to 69 years showed the highest APC $(5.4 \%)$ in participation rates for stomach cancer screening, followed by those aged 70 to $79(5.0 \%), 50$ to $59(4.3 \%)$, 


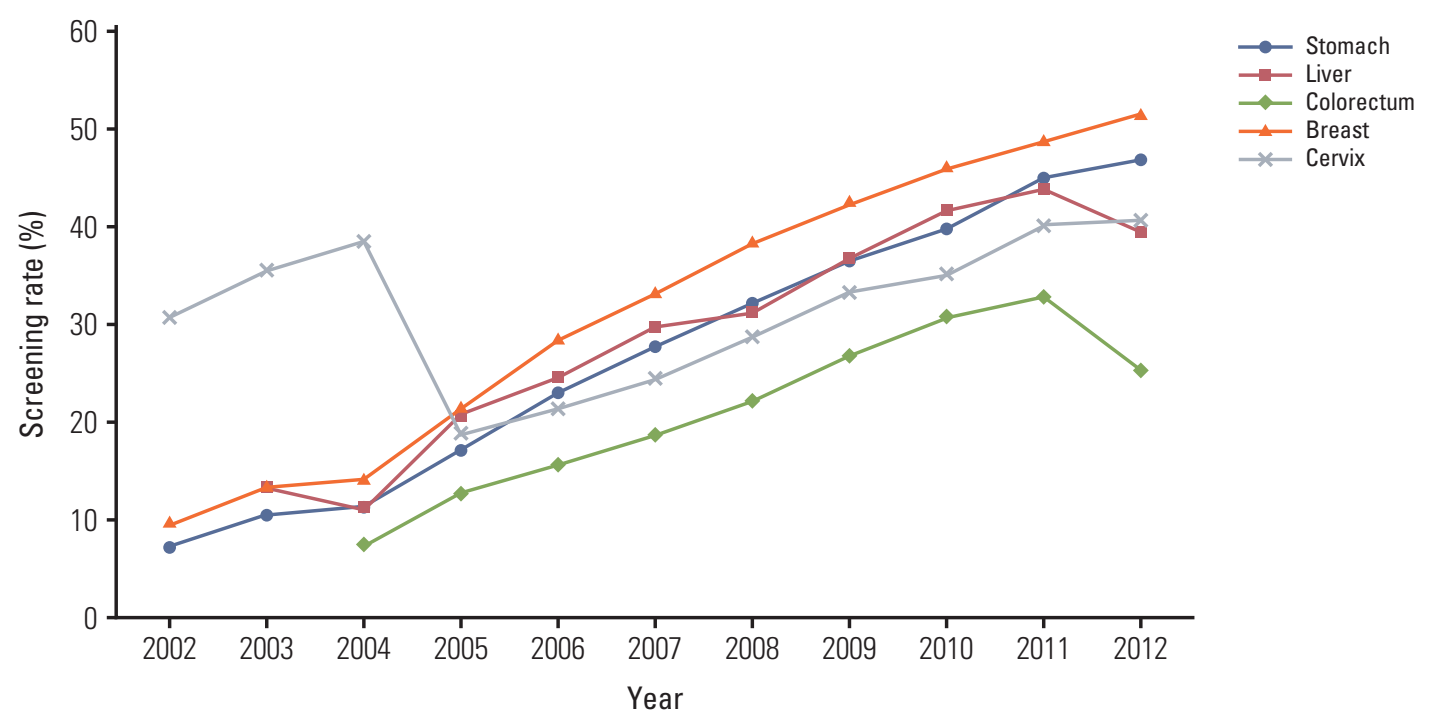

Fig. 1. Annual cancer screening rates for five major cancers from 2002 to 2012 in the National Cancer Screening Program.

and 40 to 49 years (3.8\%). Furthermore, female participants (APC, 4.6\%) and NHIS beneficiaries of higher socioeconomic status (APC, 4.9\%) showed a higher APC than average for stomach cancer screening.

Table 2 lists the participation rates for liver cancer screening. From 2003 to 2012, a total of 7.7 million men and women at high risk for liver cancer were invited to undergo screening, and 2.3 million liver cancer examinations were conducted. Participant rates increased steadily from $13.2 \%$ in 2003 to $39.5 \%$ in 2012. Individuals aged 60 to 69 years showed the highest participation rates for liver cancer screening, followed by those aged 50 to 59 and 70 to 79 years. Individuals who were NHIS beneficiaries of low-income status showed higher participation rates and the highest APC.

Between 2004 and 2012, a total of 77.8 million men and women were invited to undergo screening for colorectal cancer, and 17.6 million examinations were conducted. Participation rates for colorectal cancer gradually increased from $7.3 \%$ in 2004 to $25.0 \%$ in 2012 (Table 3). The APC in participation rates between 2004 and 2012 was 3.3\% (95\% CI, 2.5 to 4.1). An increasing tendency was observed up to 2011, while the participation rates decreased by 7.9\% from 2011 to 2012 . Individuals aged 60 to 69 years showed the highest participation rates and APC during this period (4.2\%), followed by those aged 70 to $79(3.3 \%)$ and 50 to $59(3.1 \%)$. Participation rates according to sex and health insurance status were similar.

Since inception of the breast cancer screening program, the NCSP has invited 66.3 million women to undergo breast cancer screening and supported more than 22.0 million screen- ing tests for breast cancer. The number of women served increased from 0.4 million in the first year of the breast cancer screening program to more than 3.3 million in 2012 (Table 4). Among all cancer screening programs in the NCSP, participation rates for breast cancer screening increased the most. Specifically, participation rates for breast cancer screening reached 51.9\% in 2012, up from 9.4\% in 2002. Participation rates were highest among women aged 60 to 69 years, who showed the highest APC (5.8\%), followed by women aged 50 to 59 years.

Between 2004 and 2012, a total of 77.4 million women were invited to undergo cervical cancer screening, and more than 24.6 million examinations were provided. Table 5 shows the participation rates for cervical cancer screening. Participation rates increased from 30.8\% in 2002 to $40.9 \%$ in 2012, giving an annual increase of $0.9 \%$ over the 10 -year period, which was the lowest among all cancer sites. Participation rates for cervical cancer screening were lower among MAP recipients than NHIS beneficiaries, although the MAP recipients showed a higher APC than the average. Women in their 30s showed a decreasing APC in participation rates between 2002 and 2012. Participation rates for women aged 80 years or older also decreased annually. In 2005 and 2006, there were significant decreases in participation rates for women aged 30 to 39 years. 


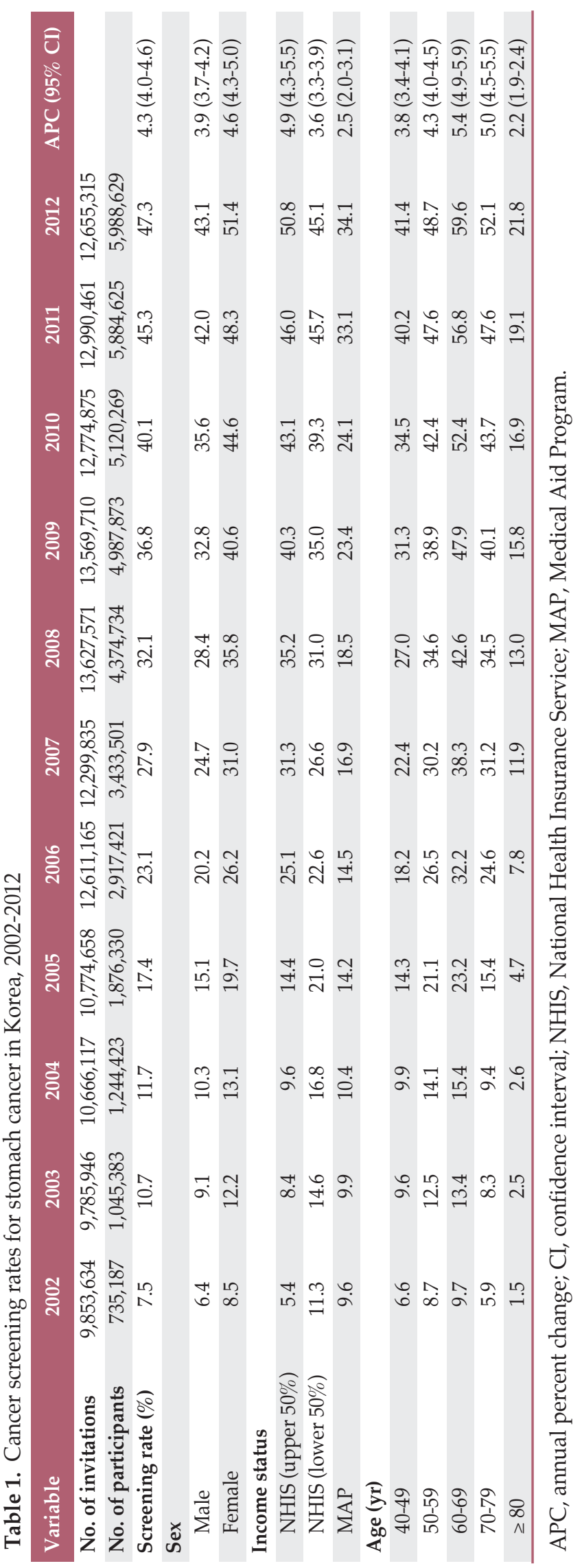




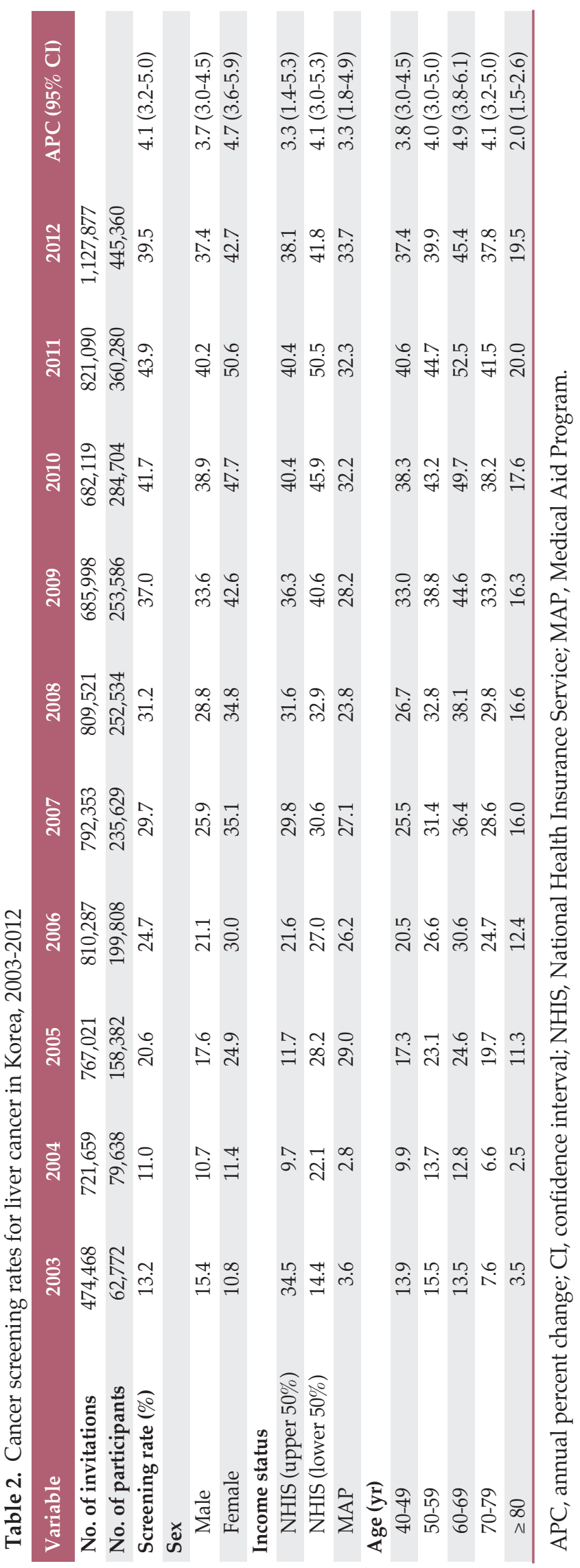




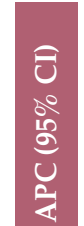

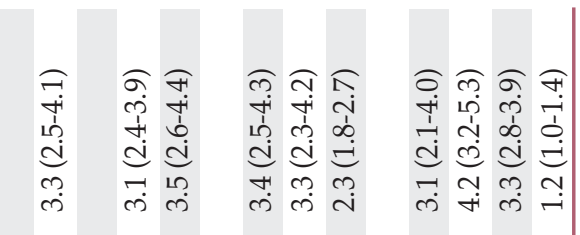

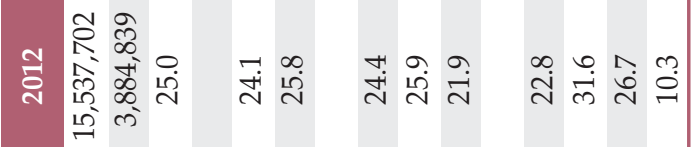

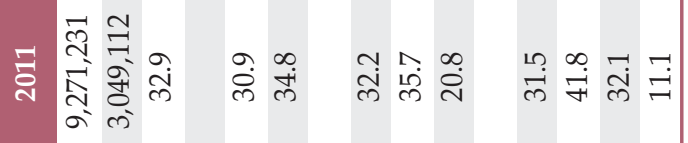

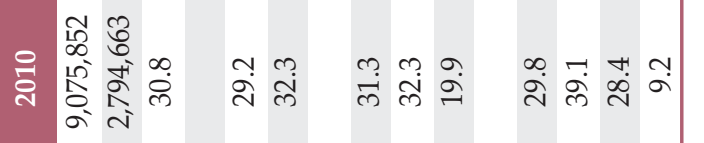

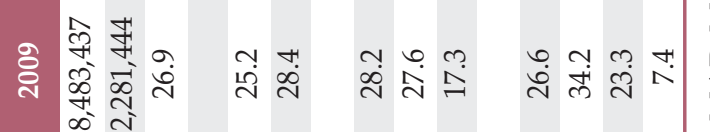

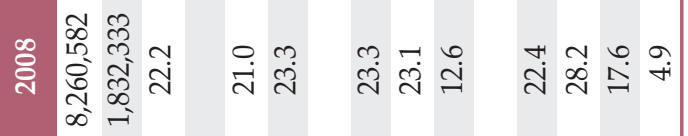

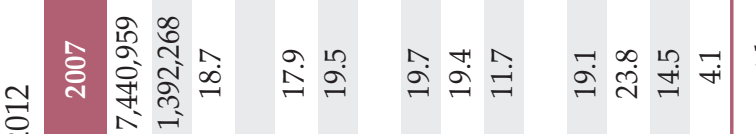

के

藏

v

ฮั

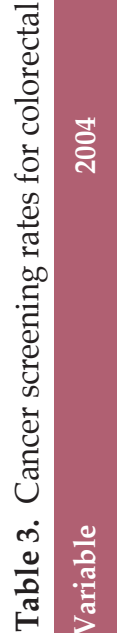

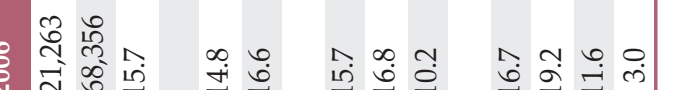
구욤

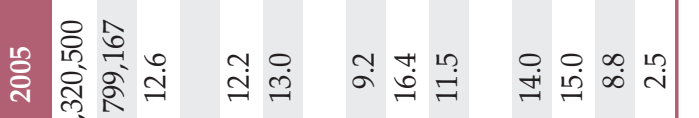

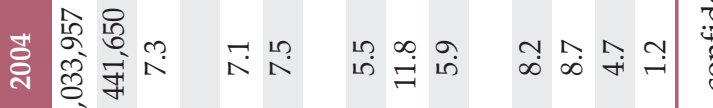

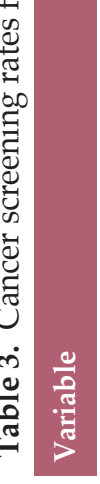

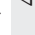

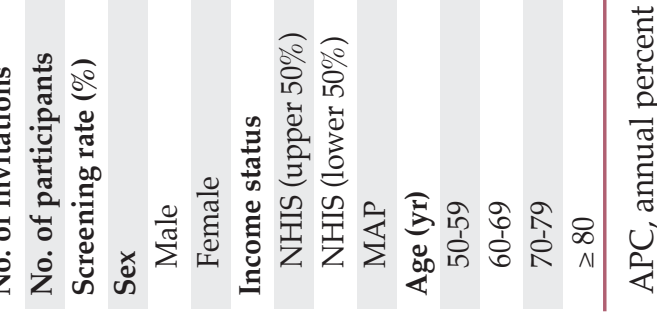

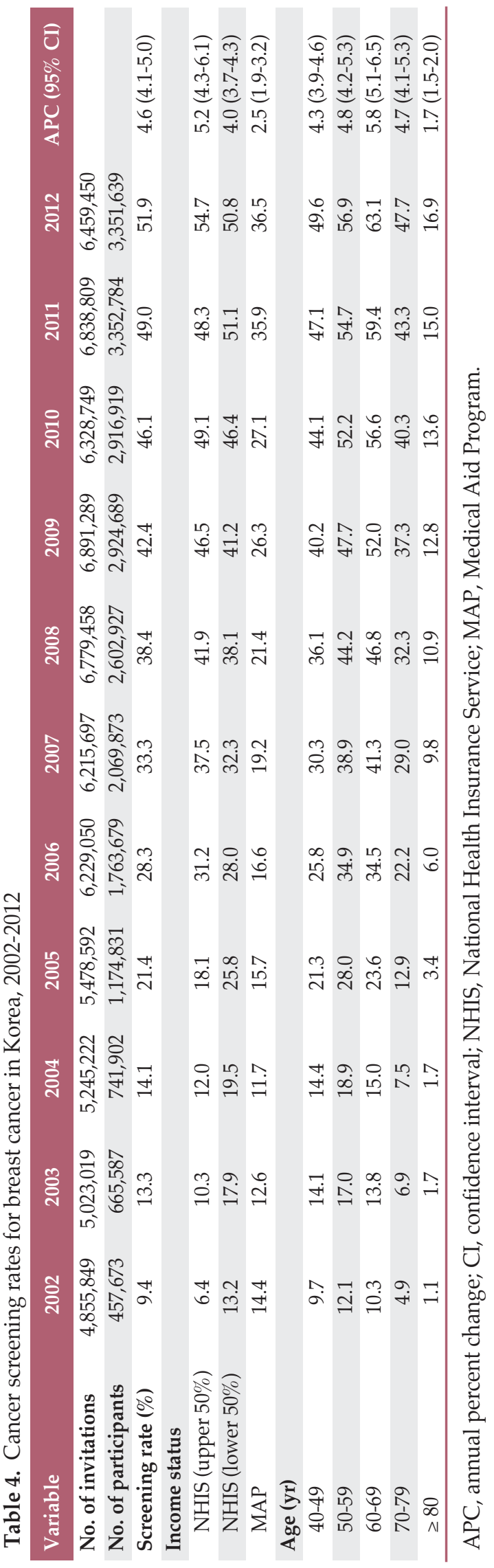




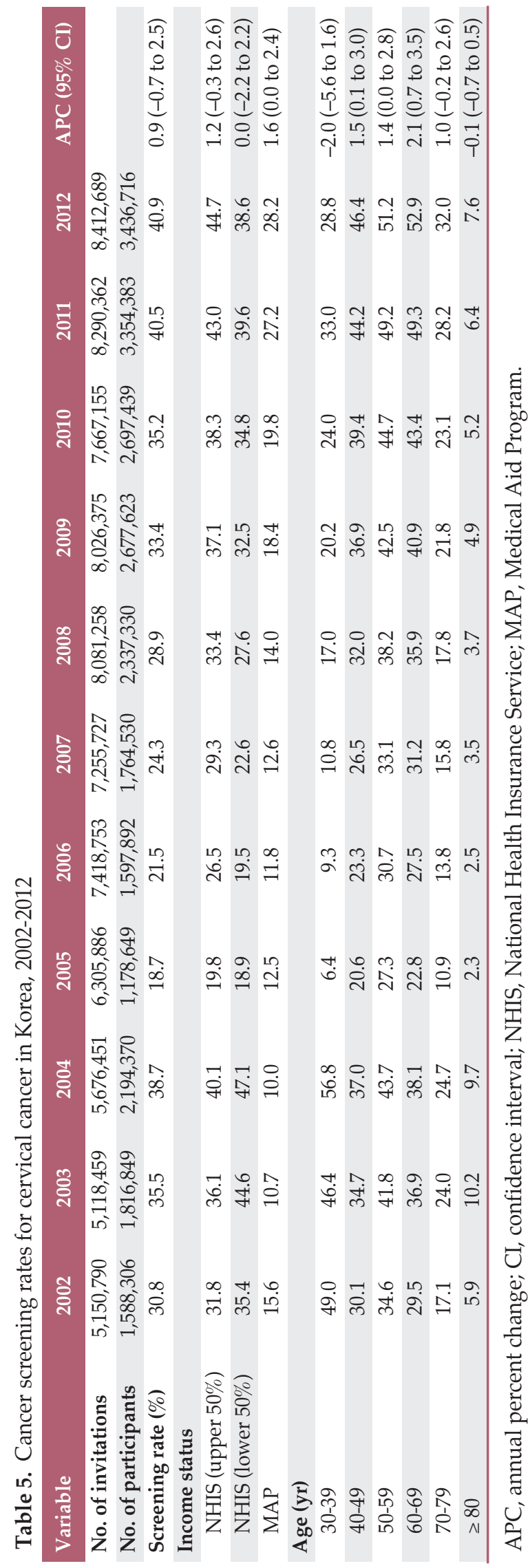

\section{Discussion}

The Korean government launched the NCSP to provide cancer screening for stomach, breast, and cervical cancer to MAP recipients in 1999. The NCSP is supported financially by the central government, local governments, and the NHIS. Up to 2001, the NCSP only offered free cancer screening to medical aid beneficiaries. By 2002, the target population of the NCSP was expanded to include NHIS beneficiaries and free-of-charge screening was expanded to include NHIS beneficiaries in the lowest $20 \%$ income stratum in 2002. Screening for liver cancer was included in 2003, and colorectal cancer screening was included in 2004. Free-of-charge screening was further expanded to include those in the lowest 50\% income stratum since 2005. Presently, MAP recipients and NHIS beneficiaries with a premium at $50 \%$ or lower are eligible for stomach, liver, colorectal, breast, and cervical cancer screening free-of-charge, while the remaining NHIS beneficiaries are eligible to undergo screening via the cancer screening program with a co-payment of $10 \%$ of the cost of the procedure [6] (S2 Table). During this period, participation rates in the NCSP for the five major cancers have increased. Participation rates showed annual increases of $4.3 \%$ for gastric cancer, $4.1 \%$ for liver cancer, 3.3\% for colorectal cancer, $4.6 \%$ for breast cancer, and $0.9 \%$ for cervical cancer. The participation rates for all cancer types, except for colorectal cancer, exceeded $40 \%$ in 2011. Breast cancer screening showed the highest participation rates, exceeding 50\% in 2012.

Despite the increasing trends in participation rates, these rates are still low relative to other nationwide cancer screening programs. In the UK, where nationwide organized cancer screening has been implemented, $73.4 \%$ of women aged 45-74 years underwent breast cancer screening by mammography in 2010-2011 [9], while 73.5\% of women aged 25-49 years underwent cervical cancer screening by Pap smear in 2011-2012 [10]. There are many possible explanations for why less than half of the target population in Korea participated in the NCSP. Various studies have identified socioeconomic and health system-related characteristics as barriers to or facilitators of cancer screening [11-14]. As one such barrier, a lack of continuity in care might contribute to lower participation rates in Korea relative to that of the UK. Previous studies have reported that general practitioners or family doctors play major roles in increasing participation rates $[15,16]$. However, in the NCSP, any clinic, hospital, or specific screening facility can apply to be certified as a cancer screening unit, and individuals who are invited to undergo screening can visit any of these certified screening units. Thus, the potential lack of a close relationship or continuous connection between the physician at the cancer screening unit and the participant might have negatively affected the 
participation rates.

The low participation rates in the NCSP might also stem from potential inconveniences associated with the screening tests. In the NCSP, participation rates for colorectal cancer screening were lowest (25\% in 2012) among the five major cancer sites. Potential reasons for the low participation rate in colorectal cancer screening might be related to screening via FOBT, which is delivered as a primary screening test in the colorectal cancer screening program in Korea. Individuals who are invited to undergo colorectal cancer screening must collect stool samples by themselves at home, and then visit a colorectal cancer screening unit to submit the sampled stool within a stool container. This process usually requires participants to visit the screening unit twice, once to pick up a stool container and again to submit the sample. This process is inconvenient to participants, and may act as a barrier to colorectal cancer screening. Therefore, efforts to reduce barriers of FOBT, such as the delivery of a stool container by mail, need to be continued, and specialized strategies according to age, sex, and region are warranted.

Our study revealed a significant drop in participation rates for cervical cancer screening in 2005. This was likely because of policy changes in the cervical cancer screening program. From 1988 to 2004, cervical cancer screening was provided through a NHIS health checkup service. In 2005, the cervical cancer screening program was separated from the checkup service and included in the NCSP. This change likely generated confusion among women who had previously underwent cervical cancer screening through the NHIS health checkup service. Furthermore, in the NCSP, invitees must voluntarily decide whether to make a screening appointment or not, while the NHIS health checkup service strongly promoted and encouraged participation. Additionally, the target population for the cervical cancer screening program was expanded to all NHIS beneficiaries over the age of 30 years in 2011. Until 2010, only MAP recipients, NHIS beneficiaries insured through their employer, and the head of a household were invited to undergo cervical cancer screening at the age of 30 years. Other subscribers and dependents were invited to undergo cervical cancer screening from the age of 40 years. In the short term, these policy changes and expansion of the target population lowered the participation rates for cervical cancer screening. Nevertheless, participation rates of cervical cancer screening began to increase again in 2006 and surpassed the previous rates in 2011. Further, the actual number of participants increased more than two times compared to the number of participants in 2002. Accordingly, the policy changes should be sufficiently publicized prior to implementation to minimize confusion.

Finally, the current study revealed trends in changes in participation rates according to socio-demographic factors. Overall, participation rates were highest for individuals aged
60-69 years, and women showed higher participation rates than men. Participation rates were lowest in underserved groups, such as MAP recipients. Moreover, the APCs in participation rates were the lowest among MAP recipients for all cancer types, except for cervical cancer, for which the screening participation rates fluctuated among NHIS beneficiaries during the study period because of policy changes to the cervical cancer screening program. There were also no significant differences in participation rates for liver and colorectal cancer screening according to socio-economic status. However, participation rates for screening of these cancer sites were too low to reveal differences according to socioeconomic status. According to previous studies, barriers to cancer screening faced by people of low socioeconomic status includes lack of time, lack of knowledge about cancer screening, physical disability or underlying disease, and logistic barriers [17]. It is possible that potential commonalities among MAP recipients (e.g., poverty and limited education) may underlie these barriers to screening. Thus, individuallytargeted interventions in a health care setting are required, such as individualized in-person or telephone counseling, individualized letters and reminders, or other individuallytargeted strategies, especially for people of lower socioeconomic status, to increase participation and reduce disparities in cancer screening.

It should be noted that our study has several limitations. The NCSP database lacks details regarding why people did not participate in the NCSP. Thus, we were unable to explore the influence of other important correlates, such as psychological factors (e.g., discomfort, concern about complications, or anxiety about the procedure) and health related factors (e.g., disability, health status, or health behaviors) that might be involved in adherence to screening. Moreover, although both organized and opportunistic cancer screenings are available in Korea, the current study used data from the NCSP database, which does not include information regarding opportunistic screening. Therefore, the results of this study should not be interpreted as reflecting overall screening rates, including both organized and opportunistic screening, for Korea. Further, the screening behaviors demonstrated in this study would not be generalizable to those for the entire Korean population.

\section{Conclusion}

This study was conducted to investigate overall trends in participation rates for stomach, liver, colorectal, breast and cervical cancer screening via the NCSP according to sex, age, and health insurance type. Overall, participation rates for all 
five cancer types continually increased from 2002 to 2012. Significant increasing trends were observed in participation rates for stomach, liver, colorectal, and breast cancer, but not for cervical cancer.

\section{Electronic Supplementary Material}

Supplementary materials are available at Cancer Research and Treatment website (http://www.e-crt.org).

\section{Conflicts of Interest}

Conflict of interest relevant to this article was not reported.

\section{Acknowledgments}

The authors thank the staff of the National Health Insurance Service. This study was supported by a Grant-in-Aid for Cancer Research and Control from the National Cancer Center of Korea (\#1610401).

\section{References}

1. Ferlay J, Soerjomataram I, Dikshit R, Eser S, Mathers C, Rebelo $\mathrm{M}$, et al. Cancer incidence and mortality worldwide: sources, methods and major patterns in GLOBOCAN 2012. Int J Cancer. 2015;136:E359-86.

2. Statistics Korea [Internet]. Daejeon: Statistics Korea; 2014 [cited 2015 Aug 6]. Available from: http:// kostat.go.kr/.

3. Jung KW, Won YJ, Kong HJ, Oh CM, Cho H, Lee DH, et al. Cancer statistics in Korea: incidence, mortality, survival, and prevalence in 2012. Cancer Res Treat. 2015;47:127-41.

4. Stewart BW, Wild CP. World cancer report 2014. Lyon: International Agency for Research on Cancer; 2014.

5. Yoo KY. Cancer control activities in the Republic of Korea. Jpn J Clin Oncol. 2008;38:327-33.

6. Kim Y, Jun JK, Choi KS, Lee HY, Park EC. Overview of the National Cancer screening programme and the cancer screening status in Korea. Asian Pac J Cancer Prev. 2011;12:725-30.

7. Hakama M. A screening programme that worked: discussion paper. J R Soc Med. 1990;83:322-4.

8. Han MA, Choi KS, Park JH, Moore MA, Park EC. Midcourse evaluation of the second-term 10-year plan for cancer control in Korea. Asian Pac J Cancer Prev. 2011;12:327-33.

9. NHS Breast Cancer Screening Programme: annual review 2012 [Internet]. Sheffield: NHS Breast Cancer Screening Programme; 2012 [cited 2015 Aug 12]. Available from: http:// www.cancerscreening.nhs.uk/breastscreen/publications/nhs bspannualreview2012.pdf.

10. NHS Cervical Cancer Screening Programme: annual review
2012 [Internet]. Sheffield: UK NHS Cancer Screening Programmes; 2012 [cited 2015 Aug 12]. Available from: http:// www.cancerscreening.nhs.uk/ cervical / publications / cervicalannual-review-2012.pdf.

11. Rakowski W, Pearlman D, Rimer BK, Ehrich B. Correlates of mammography among women with low and high socioeconomic resources. Prev Med. 1995;24:149-58.

12. Barr JK, Franks AL, Lee NC, Herther P, Schachter M. Factors associated with continued participation in mammography screening. Prev Med. 2001;33:661-7.

13. Katz SJ, Hofer TP. Socioeconomic disparities in preventive care persist despite universal coverage. Breast and cervical cancer screening in Ontario and the United States. JAMA. 1994;272:530-4.

14. Eaker S, Adami HO, Sparen P. Reasons women do not attend screening for cervical cancer: a population-based study in Sweden. Prev Med. 2001;32:482-91.

15. Lasser KE, Ayanian JZ, Fletcher RH, Good MJ. Barriers to colorectal cancer screening in community health centers: a qualitative study. BMC Fam Pract. 2008;9:15.

16. Goulard H, Boussac-Zarebska M, Ancelle-Park R, Bloch J. French colorectal cancer screening pilot programme: results of the first round. J Med Screen. 2008;15:143-8.

17. Lee YY, Jun JK, Suh M, Park BY, Kim Y, Choi KS. Barriers to cancer screening among medical aid program recipients in the Republic of Korea: a qualitative study. Asian Pac J Cancer Prev. 2014;15:589-94. 